\title{
Local Duality and Polarized Hodge Modules
}

\author{
by
}

\author{
Christian SCHNELL
}

\begin{abstract}
We find a relationship between the graded quotients of a filtered holonomic $\mathcal{D}$-module, their duals as coherent sheaves, and the characteristic variety, in case the filtered $\mathcal{D}$-module underlies a polarized Hodge module on a smooth algebraic variety. The proof is based on M. Saito's result that the associated graded module is Cohen-Macaulay, and on local duality for the cotangent bundle. The result plays a role in the study of Néron models for families of intermediate Jacobians, recently constructed by the author.
\end{abstract}

2010 Mathematics Subject Classification: Primary 32C38; Secondary 14D07, 14B15.

Keywords: polarized Hodge module, local duality, Cohen-Macaulay property, holonomicity, characteristic variety, de Rham complex.

\section{$\S 1$. Overview}

\section{$\S 1.1$. Introduction}

Filtered holonomic $\mathcal{D}$-modules play an important role in the theory of mixed Hodge modules, where they appear as natural generalizations of the flat vector bundles underlying variations of Hodge structure. One of M. Saito's main results in [7] is that a polarized variation of Hodge structure $\mathcal{H}$ on a Zariski-open subset $U$ of a complex manifold $X$ can be canonically extended to a polarized Hodge module on $X$. The extension produces, in particular, a holonomic $\mathcal{D}$-module $\mathcal{M}$ that is the minimal extension of the flat vector bundle $\left(\mathcal{H}_{\mathscr{O}}, \nabla\right)$; and a good filtration $F=F_{\bullet} \mathcal{M}$ by $\mathscr{O}_{X}$-coherent subsheaves that naturally extend the Hodge bundles, in the sense that $\left.F_{k} \mathcal{M}\right|_{U}=F^{-k} \mathcal{H}_{\mathscr{O}}$.

In general, the process that leads from $\mathcal{H}$ to the pair $(\mathcal{M}, F)$ is complicated; but there are two interesting cases where a very concrete description is possible:

Communicated by M. Kashiwara. Received December 3, 2009.

C. Schnell: Department of Mathematics, University of Illinois, Chicago, IL 60607, USA; e-mail: cschnell@math.uic.edu 
1. When $X-U$ is a divisor with normal crossing singularities, $(\mathcal{M}, F)$ is obtained from the canonical extension of the flat vector bundle $\left(\mathcal{H}_{\mathscr{O}}, \nabla\right)$ by differentiation (see $[6, \S 3.4]$, or $[8$, p. 290]).

2. When $X$ is the projective space parametrizing hypersurface sections of a smooth projective variety, and $\mathcal{H}$ is the variation of Hodge structure on the vanishing cohomology, $(\mathcal{M}, F)$ is obtained from residues of meromorphic forms, with the filtration given by pole order [10].

A recent discovery is that the sheaves $F_{k} \mathcal{M}$ can be used very nicely in geometric constructions, although they are usually not vector bundles. The best example is the author's construction of Néron models for arbitrary families of intermediate Jacobians $J(\mathcal{H}) \rightarrow U$, for $\mathcal{H}$ of weight -1 . As shown in [11], there is an analytic space $\bar{J}(\mathcal{H}) \rightarrow X$ that naturally extends the family; it is obtained as a quotient of the space $T\left(F_{0} \mathcal{M}\right)$, where for any coherent sheaf $\mathscr{F}$, we define $T(\mathscr{F})$ as the spectrum of the symmetric algebra on $\mathscr{F}$. This construction has useful consequences for the study of normal functions, especially their zero loci and singularities.

With this in mind, the purpose of this paper is to investigate the properties of the coherent sheaves $F_{k} \mathcal{M}$. The main result is a kind of duality theorem for filtered $\mathcal{D}$-modules $(\mathcal{M}, F)$ that underlie polarized Hodge modules: we show that there is a close relationship between (1) the graded quotients $\operatorname{Gr}_{k}^{F} \mathcal{M},(2)$ their duals as coherent sheaves, and (3) the characteristic variety of $\mathcal{M}$. In a forthcoming paper, we apply this seemingly technical result to show that the Néron model $\bar{J}(\mathcal{H})$ for the family of hypersurface sections of an even-dimensional smooth projective variety has many good properties.

\section{$\S 1.2$. Main result}

To state the general result, let $M$ be a polarized Hodge module of weight $w$ on a smooth complex algebraic variety $X$. There is always a Zariski-open subset $U \subseteq X$ such that the restriction of $M$ to $U$ is a polarized variation of Hodge structure of weight $n=w-d$. As above, let $(\mathcal{M}, F)$ be the filtered left holonomic $\mathcal{D}$-module underlying $M$, and let $\Theta_{X}$ be the sheaf of sections of the tangent bundle of $X$. The finitely-generated graded Sym $\Theta_{X^{-}}$module

$$
\operatorname{Gr}^{F} \mathcal{M}=\bigoplus_{p \in \mathbb{Z}} \operatorname{Gr}_{p}^{F} \mathcal{M}
$$

defines a coherent sheaf on the cotangent bundle of $X$, whose support is the characteristic variety of $\mathcal{M}$. Let $P=\mathbb{P}\left(\Theta_{X}\right)$ be the projectivization of the cotangent bundle, and $\pi: P \rightarrow X$ the natural map.

Here is the main result of this paper, stated in a way that reduces notation. 
Theorem. Let $\mathscr{C}=\mathscr{C}(\mathcal{M}, F)$ be the coherent sheaf on $P$ associated to the graded Sym $\Theta_{X}$-module $\mathscr{G}=\operatorname{Gr}^{F} \mathcal{M}$. Then for every $k \in \mathbb{Z}$, there is an exact sequence

$$
0 \rightarrow \mathcal{H o m}\left(\mathscr{G}_{-n-k}, \mathscr{O}_{X}\right) \rightarrow \mathscr{G}_{k} \rightarrow \pi_{*}\left(\mathscr{C} \otimes \mathscr{O}_{P}(k)\right) \rightarrow \mathcal{E} x t^{1}\left(\mathscr{G}_{-n-k}, \mathscr{O}_{X}\right) \rightarrow 0,
$$

where the first map is induced by the polarization on the variation of Hodge structure. For $i \geq 2$ and $k \in \mathbb{Z}$, we similarly have isomorphisms

$$
R^{i-1} \pi_{*}\left(\mathscr{C} \otimes \mathscr{O}_{P}(k)\right) \simeq \mathcal{E} x t^{i}\left(\mathscr{G}_{-n-k}, \mathscr{O}_{X}\right)
$$

Of course, there are more elegant versions: one really has an exact sequence and isomorphisms of graded $\mathscr{S}$-modules, where $\mathscr{S}=$ Sym $\Theta_{X}$ (see Theorem 3.2); and, in fact, they come from a triangle in a suitable derived category (see Theorem 4.3).

A special case of the theorem was obtained in [10] for the family of hypersurface sections of a smooth projective variety, by lengthy calculations (using the description of $(\mathcal{M}, F)$ in terms of residues). Here, we give a far more conceptual proof for the general result, based on two ingredients: a result by Saito and Kashiwara, to the effect that $\operatorname{Gr}^{F} \mathcal{M}$ is Cohen-Macaulay as an $\mathscr{S}$-module when $(\mathcal{M}, F)$ underlies a polarized Hodge module (see [9, p. 55] for more information); and local duality on the cotangent bundle $T_{X}^{*}$, relative to the zero section.

To orient the reader, here is a brief outline of the paper: After recalling the relevant facts about local cohomology and local duality in $\$ 2.1-2.2$, we deduce our main result from the Cohen-Macaulay property of $\operatorname{Gr}^{F} \mathcal{M}$ in $\S 3.1-3.2$. We also show, in $\S 3.3$, that the initial map in the exact sequence is induced by the polarization of $M$. Concretely, this means that on the dense open set where $M$ is a polarized variation of Hodge structure of weight $n$, the map

$$
\mathcal{H o m}_{\mathscr{O}_{X}}\left(\mathscr{G}_{-n-k}, \mathscr{O}_{X}\right) \rightarrow \mathscr{G}_{k}
$$

is given (up to a sign factor) by $S(-,-)$, for $S$ the polarization and $d=\operatorname{dim} X$. As mentioned above, the exact sequence and the isomorphisms in Theorem 3.2 are really part of an exact triangle in the derived category (of quasi-coherent graded $\mathscr{S}$-modules). This circumstance is useful when applying other functors, and so we deduce it from the preceding sections in $\S 4.1$.

\section{§1.3. Applications}

The main application of Theorem 3.2 is to the study of the spaces $T\left(F_{k} \mathcal{M}\right)$, and hence to the Néron model of [11]. We briefly describe the result; more details can be found in [12]. Suppose that $X=\left|\mathscr{O}_{Y}(m)\right|$ parametrizes hypersurface sections of degree $m$ of a smooth projective variety $Y$ of dimension $n+1$. Denote by 
$\pi: \mathscr{Y} \rightarrow X$ the corresponding family of hypersurfaces $\mathscr{Y}_{x}=\pi^{-1}(x)$. Let $\mathcal{H}$ be the variation of Hodge structure with fibers the variable part of the cohomology,

$$
\mathcal{H}_{x}=\operatorname{ker}\left(H^{n}\left(\mathscr{Y}_{x}, \mathbb{Q}\right) \rightarrow H^{n+2}(X, \mathbb{Q}(1))\right),
$$

and let $(\mathcal{M}, F)$ be the filtered $\mathcal{D}$-module underlying its extension to a polarized Hodge module on $X$.

Theorem. Given an integer $p>0$, the sheaves $F_{k} \mathcal{M}$ in the range $-n \leq k \leq 0$ satisfy Serre's condition $S_{p}$ once $m \gg 0$. In particular, they are reflexive.

It follows that the Néron model $\bar{J}(\mathcal{H})$ (for odd values of $n$ ) is, except over a subset whose codimension increases with $m$, the quotient of a vector bundle; in particular, it is a complex manifold in a neighborhood of those points.

To verify Serre's condition, one has to show that the support of the sheaves $\mathcal{E} x t^{i}\left(\mathscr{G}_{k}, \mathscr{O}_{X}\right)$ has codimension at least $i+p$, for every $i>0$. This can be done with the help of Theorem 3.2, as follows: First, one proves that the family of hypersurface sections is naturally embedded into the projectivized cotangent bundle $P$, and that the support of the sheaf $\mathscr{C}(\mathcal{M}, F)$ is precisely the union of all the singular points in the hypersurfaces. By taking $m \gg 0$, one can make the codimension of the subset of $X$ corresponding to hypersurfaces with "many" singularities arbitrarily large and deduce the necessary vanishing of the $\mathcal{E} x t$-sheaves from the theorem.

Another consequence is the following vanishing theorem for $\mathcal{E} x t$-sheaves.

Theorem. If the hypersurface $\mathscr{Y}_{x}$ corresponding to a point $x \in X$ has a singular locus of dimension $\leq k$, then the coherent sheaves $\mathcal{E} x t^{i}\left(\mathscr{G}_{k}, \mathscr{O}_{X}\right)$ vanish in a neighborhood of $x$ for all $i \geq k+1$.

In $\S 4.3$, we also give a small application of Theorem 3.2 to the study of the de Rham complex $\operatorname{DR}_{X}(\mathcal{M})$, where $(\mathcal{M}, F)$ is still a filtered left $\mathcal{D}$-module underlying a polarized Hodge module $M$ of weight $n+d$. The complex is naturally filtered by subcomplexes $F_{k} \mathrm{DR}_{X}(\mathcal{M})$, and we show that the inclusion $F_{p-n-1} \operatorname{DR}_{X}(\mathcal{M}) \subseteq$ $\operatorname{DR}_{X}(\mathcal{M})$ is a filtered quasi-isomorphism, where $p \in \mathbb{Z}$ is such that $F_{-p} \mathcal{M}=0$. For example, when $M$ is the intermediate extension of a polarized variation of Hodge structure of weight $n$, and $F^{n+1} \mathcal{M}=F_{-n-1} \mathcal{M}=0$, then $F_{0} \operatorname{DR}_{X}(\mathcal{M}) \subseteq$ $\operatorname{DR}_{X}(\mathcal{M})$ is a quasi-isomorphism. This fact played a role in [10], where properties of $(\mathcal{M}, F)$ were used to study normal functions associated to primitive Hodge classes.

\section{§1.4. Notation}

We will use the following notation when working with sheaves of graded modules. For $E \rightarrow X$ a vector bundle of rank $d$, and $\mathscr{E}$ the corresponding locally free sheaf 
on $X$, we let $\mathscr{S}=\operatorname{Sym} \mathscr{E}^{\vee}$ be the symmetric algebra. We define

$$
\omega_{\mathscr{S}}=\operatorname{det} \mathscr{E}^{\vee} \otimes_{\mathscr{O}_{X}} \mathscr{S}(-d) .
$$

Let $\mathscr{G}$ be a graded $\mathscr{S}$-module. Its graded dual is the module

$$
D(\mathscr{G})=\bigoplus_{k \in \mathbb{Z}} \mathcal{H o m}_{\mathscr{O}_{X}}\left(\mathscr{G}_{-k}, \mathscr{O}_{X}\right)
$$

with $\mathcal{H o m}\left(\mathscr{G}_{-k}, \mathscr{O}_{X}\right)$ in degree $k$, and the module structure given by the rule $(s \phi)(g)=\phi(s g)$. The involution $(-1): E \rightarrow E$ allows us to define

$$
\mathscr{G}^{\mathrm{r}}=(-1)^{*} \mathscr{G}
$$

this is the same graded $\mathscr{O}_{X}$-module as $\mathscr{G}$, but with the $\mathscr{S}$-module structure changed so that sections of $\mathscr{S}_{k}$ act with an extra factor of $(-1)^{k}$. When $\mathscr{G}$ is $d$ dimensional and Cohen-Macaulay as an $\mathscr{S}$-module, meaning that $\mathcal{E}^{i} t_{\mathscr{S}}(\mathscr{G}, \mathscr{S})=0$ for $i \neq d$, we call the sheaf

$$
\widehat{\mathscr{G}}=\mathcal{E x t}_{\mathscr{S}}^{d}\left(\mathscr{G}, \omega_{\mathscr{S}}\right)
$$

the dual $\mathscr{S}$-module.

Except where mentioned otherwise, we use left $\mathcal{D}$-modules in this paper (where [8] uses right $\mathcal{D}$-modules). When $M$ is a mixed Hodge module, the effect of a Tate twist $M(k)$ on the underlying filtered $\mathcal{D}$-module $(\mathcal{M}, F)$ is as follows: $(\mathcal{M}, F)(k)=$ $\left(\mathcal{M}, F_{\bullet-k}\right)$.

\section{$\S 2$. Local duality on vector bundles}

Let $X$ be a smooth algebraic variety (or any quasi-compact variety where every coherent sheaf is the quotient of a locally free one), and $E \rightarrow X$ a vector bundle of rank $d \geq 1$. We review several facts about local cohomology on $E$ with support in the zero section, as well as about local duality. The case when $E$ is an affine space is well-known, and is explained very clearly in Appendix 1 of D. Eisenbud's book [3, pp. 187-199]. Short proofs are included here for the sake of completeness; they are mostly straightforward generalizations of the ones in [3].

\section{$\S 2.1$. Local cohomology on a vector bundle}

Let $\mathscr{E}$ be a locally free sheaf on $X$ of rank $d \geq 1$. The symmetric algebra

$$
\mathscr{S}=\operatorname{Sym} \mathscr{E}^{\vee}=\bigoplus_{k \geq 0} \operatorname{Sym}^{k} \mathscr{E} \vee
$$

is a sheaf of graded $\mathscr{O}_{X}$-algebras, and $E=\operatorname{Spec} \mathscr{S}$ is the vector bundle corresponding to $\mathscr{E}$. The map $f: E \rightarrow X$ is affine, and we have $\mathscr{S} \simeq f_{*} \mathscr{O}_{E}$. Quasi-coherent 
sheaves on $E$ are in one-to-one correspondence with quasi-coherent $\mathscr{S}$-modules on $X$; given a sheaf of $\mathscr{S}$-modules $\mathscr{G}$, we let $\mathscr{G}_{E}$ be the corresponding sheaf on $E$, so that $f_{*} \mathscr{G}_{E} \simeq \mathscr{G}$.

The original variety $X$ is naturally embedded into $E$ by the zero section of the vector bundle. Let $\mathscr{F}$ be any quasi-coherent sheaf on $E$. The subsheaf $\mathcal{H}_{X}^{0}(\mathscr{F})$ consists of all sections of $\mathscr{F}$ whose support is contained in the zero section. Then $\mathcal{H}_{X}^{0}$ is a left-exact functor on quasi-coherent $\mathscr{O}_{E}$-modules, and its $i$-th right-derived functor is denoted by $\mathcal{H}_{X}^{i}$; we call the sheaf $\mathcal{H}_{X}^{i}(\mathscr{F})$ the $i$-th local cohomology sheaf of $\mathscr{F}$ with support in the zero section of $E$. The corresponding quasi-coherent $\mathscr{S}$ module is $f_{*} \mathcal{H}_{X}^{i}(\mathscr{F})$; when $\mathscr{G}$ is a graded $\mathscr{S}$-module, the local cohomology modules $f_{*} \mathcal{H}_{X}^{i}\left(\mathscr{G}_{E}\right)$ are naturally graded $\mathscr{S}$-modules as well. More information about local cohomology sheaves can be found in [5, Exposés I and II on pp. 5-26], in the expected greater generality.

We also consider the projectivization of the vector bundle, given by $P=$ Proj $\mathscr{S}$, together with the projection map $\pi: P \rightarrow X$ (see [4, Chapitre II, §3] for details). As usual, we write $\mathscr{O}_{P}(1)$ for the universal line bundle on $P$. A finitely generated graded $\mathscr{S}$-module

$$
\mathscr{G}=\bigoplus_{k \in \mathbb{Z}} \mathscr{G}_{k}
$$

defines a coherent sheaf $\mathscr{G}_{P}$ on the projective bundle $P$. We let $\mathscr{G}(m)$ be the graded $\mathscr{S}$-module with $\mathscr{G}(m)_{k}=\mathscr{G}_{m+k}$; evidently, $\mathscr{O}_{P}(1)$ is the coherent sheaf associated to $\mathscr{S}(1)$. For $\mathscr{F}$ a coherent sheaf on $P$, and $i \geq 0$, we have a graded $\mathscr{S}$-module

$$
R^{i} \Gamma_{*}(\mathscr{F})=\bigoplus_{k \in \mathbb{Z}} R^{i} \pi_{*}\left(\mathscr{F} \otimes \mathscr{O}_{P}(k)\right)
$$

we usually write $\Gamma_{*}(\mathscr{F})$ in place of $R^{0} \Gamma_{*}(\mathscr{F})$. Since $\mathscr{O}_{P}(1)$ is relatively ample, the natural map $\mathscr{G} \rightarrow \Gamma_{*}\left(\mathscr{G}_{P}\right)$ is an isomorphism in large degrees; its behavior in arbitrary degrees is related to the local cohomology sheaves of $\mathscr{G}_{E}$, as shown by the following proposition.

Proposition 2.1. Let $\mathscr{G}$ be a graded $\mathscr{S}$-module on $X$. With the notation introduced above, there is an exact sequence

$$
0 \rightarrow f_{*} \mathcal{H}_{X}^{0}\left(\mathscr{G}_{E}\right) \rightarrow \mathscr{G} \rightarrow \Gamma_{*}\left(\mathscr{G}_{P}\right) \rightarrow f_{*} \mathcal{H}_{X}^{1}\left(\mathscr{G}_{E}\right) \rightarrow 0
$$

of graded $\mathscr{S}$-modules. Moreover, for each $i \geq 2$, we have an isomorphism

$$
R^{i-1} \Gamma_{*}\left(\mathscr{G}_{P}\right) \simeq f_{*} \mathcal{H}_{X}^{i}\left(\mathscr{G}_{E}\right),
$$

again of graded $\mathscr{S}$-modules. 
Proof. For the convenience of the reader, we briefly review the argument. Consider the following commutative diagram of maps:

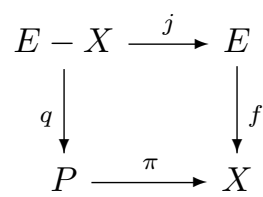

By [5, Corollaire 2.11], we have an exact sequence

$$
0 \rightarrow \mathcal{H}_{X}^{0}\left(\mathscr{G}_{E}\right) \rightarrow \mathscr{G}_{E} \rightarrow j_{*} j^{*} \mathscr{G}_{E} \rightarrow \mathcal{H}_{X}^{1}\left(\mathscr{G}_{E}\right) \rightarrow 0 .
$$

Now $\mathscr{G}$ is graded, and so we have $j^{*} \mathscr{G}_{E} \simeq q^{*} \mathscr{G}_{P}$. Using the projection formula, we then find that

$$
f_{*} j_{*} j^{*} \mathscr{G}_{E} \simeq \pi_{*}\left(\mathscr{G}_{P} \otimes q_{*} \mathscr{O}_{E-X}\right) \simeq \pi_{*}\left(\mathscr{G}_{P} \otimes \bigoplus_{k \in \mathbb{Z}} \mathscr{O}_{P}(k)\right) \simeq \Gamma_{*}\left(\mathscr{G}_{P}\right) .
$$

Applying the exact functor $f_{*}$ to the sequence in $(2.2)$, and noting that $f_{*} \mathscr{G}_{E} \simeq \mathscr{G}$, we obtain the first half of the proposition. The second half follows by similar considerations from the isomorphism $R^{i-1} j_{*} j^{*} \mathscr{G}_{E} \simeq \mathcal{H}_{X}^{i}\left(\mathscr{G}_{E}\right)$ for $i \geq 2$, also given in $[5$, p. 16].

\section{$\S 2.2$. Local duality on a vector bundle}

Given a graded $\mathscr{S}$-module $\mathscr{G}$, we define its graded dual to be

$$
D(\mathscr{G})=\bigoplus_{k \in \mathbb{Z}} \mathcal{H o m}_{\mathscr{O}_{X}}\left(\mathscr{G}_{-k}, \mathscr{O}_{X}\right)
$$

This is again a graded $\mathscr{S}$-module, with the summand $\mathcal{H}_{m} \mathscr{O}_{X}\left(\mathscr{G}_{-k}, \mathscr{O}_{X}\right)$ in degree $k$; the action of $\mathscr{S}$ is given by the rule $(s \cdot \phi)(g)=\phi(s g)$. The $i$-th derived functor of $D$ is then evidently

$$
D^{i}(\mathscr{G})=\bigoplus_{k \in \mathbb{Z}} \mathcal{E} x t_{\mathscr{O}_{X}}^{i}\left(\mathscr{G}_{-k}, \mathscr{O}_{X}\right)
$$

Note that even when $\mathscr{G}$ is finitely generated as an $\mathscr{S}$-module, $D^{i}(\mathscr{G})$ is usually not; unless, of course, $\mathscr{G}$ actually has finite length.

In analogy with the canonical line bundle on projective space, we also introduce the graded $\mathscr{S}$-module $\omega_{\mathscr{S}}=\operatorname{det} \mathscr{E}^{\vee} \otimes_{\mathscr{O} X} \mathscr{S}(-d)$, whose graded piece in degree $k$ is $\operatorname{det} \mathscr{E}^{\vee} \otimes \mathrm{Sym}^{k-d} \mathscr{E}^{\vee}$; here $d$ is still the rank of the vector bundle. Then $\omega_{P / X}$ is the sheaf associated to $\omega_{\mathscr{S}}$, because a simple calculation with the Euler sequence for $\pi: P \rightarrow X$ shows that

$$
\omega_{P / X} \simeq\left(\pi^{*} \operatorname{det} \mathscr{E}^{\vee}\right) \otimes \mathscr{O}_{P}(-d)
$$


The second important result about local cohomology sheaves on a vector bundle is the following duality theorem, known as graded local duality in the case of an affine space.

Proposition 2.5. Let $\mathscr{G}$ be a finitely generated graded $\mathscr{S}$-module on $X$. Then there is a convergent fourth-quadrant spectral sequence of graded $\mathscr{S}$-modules,

$$
E_{2}^{p, q}=D^{p}\left(\mathcal{E}_{x} t_{\mathscr{S}}^{-q}\left(\mathscr{G}, \omega_{\mathscr{S}}\right)\right) \Rightarrow \mathcal{H}_{X}^{d+p+q}\left(\mathscr{G}_{E}\right)
$$

functorial in the sheaf $\mathscr{G}$.

The following notion will be useful during the proof. A graded $\mathscr{S}$-module is called basic if it is a finite direct sum of modules of the form $\mathscr{B} \otimes_{\mathscr{O}_{X}} \mathscr{S}(m)$, with $\mathscr{B}$ a locally free $\mathscr{O}_{X}$-module. The local cohomology sheaves are easy to describe in that case.

Lemma 2.6. Let $\mathscr{F}=\mathscr{B} \otimes \mathscr{S}(m)$ be a basic graded $\mathscr{S}$-module. Then

$$
f_{*} \mathcal{H}_{X}^{i}\left(\mathscr{F}_{E}\right) \simeq \begin{cases}D\left(\mathcal{H o m}_{\mathscr{S}}\left(\mathscr{F}, \omega_{\mathscr{S}}\right)\right) & \text { for } i=d, \\ 0 & \text { otherwise } .\end{cases}
$$

Proof. Since $\mathscr{B}$ is locally free, one is quickly reduced to the case $\mathscr{F}=\mathscr{S}$, where then $\mathscr{F}_{E}=\mathscr{O}_{E}$. When $d=1$, the assertion follows immediately from the exact sequence in Proposition 2.1. Thus we may assume from now on that $d \geq 2$. Since $\pi_{*} \mathscr{O}_{P}(k) \simeq \mathscr{S}_{k}$, while $R^{i} \pi_{*} \mathscr{O}_{P}(k)=0$ for $1 \leq i \leq d-1$, Proposition 2.1 shows that $\mathcal{H}_{X}^{i}\left(\mathscr{O}_{E}\right)=0$ for $i \neq d$. For $i=d$, we let

$$
\mathcal{H}=f_{*} \mathcal{H}_{X}^{d}\left(\mathscr{O}_{E}\right) \simeq \bigoplus_{k \in \mathbb{Z}} R^{d-1} \pi_{*} \mathscr{O}_{P}(k)
$$

At this point, one can easily obtain the isomorphism in (2.7) by using duality for the morphism $\pi: P \rightarrow X$. Following [3, p. 191], we shall give a more concrete derivation using a Čech complex, because this has the advantage of showing the $\mathscr{S}$-module structure on $\mathcal{H}$ more clearly.

We first consider the problem locally. So let $U \simeq \operatorname{Spec} A$ be an affine open subset of $X$ over which the vector bundle $E$ is trivial. Then $S=\Gamma(U, \mathscr{S}) \simeq$ $A\left[t_{1}, \ldots, t_{d}\right]$ as graded $A$-algebras, and $f^{-1}(U) \simeq \operatorname{Spec} S$. Let $I=A t_{1}+\cdots+A t_{d}$ be the irrelevant ideal, and $V(I)=\operatorname{Spec}(S / I)$. As in the proof of Proposition 2.1, the local cohomology module $H=\Gamma(U, \mathcal{H})$ that we need to compute is

$$
H=H_{V(I)}^{d}(\operatorname{Spec} S, \mathscr{O}) \simeq H^{d-1}(\operatorname{Spec} S-V(I), \mathscr{O})
$$


it can be found by the Čech complex for the standard open cover of Spec $S-V(I)$. As a graded $S$-module, $H$ is therefore isomorphic to the cokernel of the map

$$
\bigoplus_{i=1}^{d} S\left[\left(t_{1} \cdots t_{i-1} t_{i+1} \cdots t_{d}\right)^{-1}\right] \rightarrow S\left[\left(t_{1} \cdots t_{d}\right)^{-1}\right] .
$$

Thus $H_{k}$ is generated by elements of the form $F /\left(t_{1} \cdots t_{d}\right)^{m}$, where $F$ is a homogeneous polynomial of degree $k+d m$. From degree considerations, we see that $H_{k}=0$ for $k>-d$, while $H_{-d} \simeq A$, generated by $\left(t_{1} \cdots t_{d}\right)^{-1}$. We now have a map

$$
H_{k} \rightarrow \operatorname{Hom}_{A}\left(S_{-k-d}, H_{-d}\right),
$$

by sending an element $F /\left(t_{1} \cdots t_{d}\right)^{m} \in H_{k}$ to the functional $G \mapsto F G /\left(t_{1} \cdots t_{d}\right)^{m}$, for $G \in S_{-k-d}$. This is easily seen to be an isomorphism; moreover, since the $S$-module structure on $H$ is simply given by multiplication, we obtain

$$
H \simeq \bigoplus_{k \in \mathbb{Z}} \operatorname{Hom}_{A}\left(S_{-k-d}, H_{-d}\right)=H_{-d} \otimes_{A} D(S(-d))
$$

as graded $S$-modules. As written, the isomorphism is coordinate-independent, and so we get a global isomorphism of graded $\mathscr{S}$-modules

$$
\mathcal{H} \simeq \mathcal{H}_{-d} \otimes_{\mathscr{O}_{X}} D(\mathscr{S}(-d)) .
$$

Note that $\mathcal{H}_{-d}$ has rank one, and is therefore a line bundle on $X$. Because of (2.8), we find that $\mathcal{H}_{-d} \simeq R^{d-1} \pi_{*} \mathscr{O}_{P}(-d) \simeq \operatorname{det} \mathscr{E}$, and this concludes the proof.

Lemma 2.9. Let $\mathscr{G}$ be a finitely generated graded $\mathscr{S}$-module on $X$. Then $\mathscr{G}$ can be resolved in the form

$$
\cdots \rightarrow \mathscr{F}^{-2} \rightarrow \mathscr{F}^{-1} \rightarrow \mathscr{F}^{0} \rightarrow \mathscr{G} \rightarrow 0
$$

by basic graded $\mathscr{S}$-modules $\mathscr{F}^{i}$.

Proof. It suffices to show that every finitely generated graded $\mathscr{S}$-module $\mathscr{G}$ admits a surjection from a basic one. Since $\mathscr{G}$ is finitely generated, and $X$ is quasi-compact, there is a finite set $F \subseteq \mathbb{Z}$ such that

$$
\bigoplus_{k \in F} \mathscr{G}_{k} \otimes_{\mathscr{O}_{X}} \mathscr{S}(-k) \rightarrow \mathscr{G}
$$

is surjective. Each $\mathscr{G}_{k}$ is a coherent sheaf of $\mathscr{O}_{X}$-modules, and because $X$ is smooth, there is a locally free sheaf $\mathscr{B}_{k}$ mapping onto $\mathscr{G}_{k}$. Then $\mathscr{F}^{0}=\bigoplus_{k \in F} \mathscr{B}_{k} \otimes \mathscr{S}(-k)$ is a basic module mapping onto $\mathscr{G}$.

Here is the proof of Proposition 2.5. 
Proof. Let $\mathscr{G}$ be any finitely generated graded $\mathscr{S}$-module. According to Lemma 2.9 , there is a complex $\mathscr{F}^{\bullet}$ of basic graded $\mathscr{S}$-modules resolving $\mathscr{G}$. The local cohomology sheaves of $\mathscr{G}$ are therefore computed by a spectral sequence

$$
E_{1}^{p, q}=f_{*} \mathcal{H}_{X}^{q}\left(\mathscr{F}^{p}\right) \Rightarrow \mathcal{H}_{X}^{p+q}\left(\mathscr{G}_{E}\right)
$$

From (2.7), all but one row of the $E_{1}$-page is zero, and so $\mathcal{H}_{X}^{d+i}\left(\mathscr{G}_{E}\right)$ is isomorphic to the cohomology in degree $i$ of the complex

$$
f_{*} \mathcal{H}_{X}^{d}\left(\mathscr{F}^{\bullet}\right) \simeq D\left(\mathcal{H o m}_{\mathscr{S}}\left(\mathscr{F}^{\bullet}, \omega_{\mathscr{S}}\right)\right) .
$$

The spectral sequence is now simply the one for the composition of the two contravariant functors $\mathcal{H o m}_{\mathscr{S}}\left(-, \omega_{\mathscr{S}}\right)$ and $D$.

\section{§2.3. Local duality for Cohen-Macaulay modules}

Now suppose that the graded $\mathscr{S}$-module $\mathscr{G}$ is in addition Cohen-Macaulay of dimension $d$; that is, the associated coherent sheaf $\mathscr{G}_{E}$ is Cohen-Macaulay on $E$, with purely $d$-dimensional support. Consequently, $\mathcal{E}^{q} t_{\mathscr{S}}^{q}(\mathscr{G}, \mathscr{S})=0$ unless $q=d$; let

$$
\widehat{\mathscr{G}}=\mathcal{E}_{x} t_{\mathscr{S}}^{d}\left(\mathscr{G}, \omega_{\mathscr{S}}\right)=\mathcal{E}_{x} t_{\mathscr{S}}^{d}(\mathscr{G}, \mathscr{S}(-d)) \otimes_{\mathscr{O}_{X}} \operatorname{det} \mathscr{E}^{\vee}
$$

be the dual $\mathscr{S}$-module. The spectral sequence in Proposition 2.5 degenerates at the $E_{2}$-page, because it has only one nonzero row, and we find that

$$
f_{*} \mathcal{H}_{X}^{p}\left(\mathscr{G}_{E}\right) \simeq D^{p}(\widehat{\mathscr{G}})=\bigoplus_{k \in \mathbb{Z}} \mathcal{E} x t_{\mathscr{O}_{X}}^{p}\left(\widehat{\mathscr{G}}_{-k}, \mathscr{O}_{X}\right)
$$

for all $p \geq 0$. In combination with Proposition 2.1, we now get the following result.

Theorem 2.10. Let $\mathscr{G}$ be a finitely generated graded $\mathscr{S}$-module, which is CohenMacaulay of dimension $d=\operatorname{rk} \mathscr{E}$. Let $\widehat{\mathscr{G}}=\mathcal{E} t_{\mathscr{S}}^{d}\left(\mathscr{G}, \omega_{\mathscr{S}}\right)$ be the dual module. Then there is an exact sequence

$$
0 \rightarrow D(\widehat{\mathscr{G}}) \rightarrow \mathscr{G} \rightarrow \Gamma_{*}\left(\mathscr{G}_{P}\right) \rightarrow D^{1}(\widehat{\mathscr{G}}) \rightarrow 0
$$

of graded $\mathscr{S}$-modules. Moreover, for each $i \geq 2$, we have an isomorphism

$$
R^{i-1} \Gamma_{*}\left(\mathscr{G}_{P}\right) \simeq D^{i}(\widehat{\mathscr{G}}),
$$

again respecting the graded $\mathscr{S}$-module structure on both sides.

\section{§3. Polarized Hodge modules}

From now on, let $X$ be a nonsingular complex algebraic variety of dimension $d \geq 1$. The cotangent bundle $E=T_{X}^{*}$ is then a vector bundle of rank $d$ on $X$; as in $\S 2.1$, 
we let $P=\mathbb{P}\left(\Theta_{X}\right)$ be its projectivization, $\pi: P \rightarrow X$ the natural map, and $\mathscr{O}_{P}(1)$ the universal line bundle on $P$. Also let $\mathscr{S}=\operatorname{Sym} \Theta_{X}$. Note that $\operatorname{det} \mathscr{E}=\omega_{X}$, and so $\omega_{\mathscr{S}}=\omega_{X}^{-1} \otimes_{\mathscr{O}_{X}} \mathscr{S}(-d)$.

\section{$\S 3.1$. The Cohen-Macaulay property}

Let $M$ be a polarized Hodge module on $X$ of weight $w$ (see [9] for an overview of the theory). We write $(\mathcal{M}, F)$ for the underlying filtered holonomic left $\mathcal{D}$-module, and consider the graded $\mathscr{S}$-module

$$
\mathscr{G}=\mathrm{Gr}^{F} \mathcal{M}=\bigoplus_{k \in \mathbb{Z}} \operatorname{Gr}_{k}^{F} \mathcal{M}
$$

As before, $\mathscr{G}_{E}$ denotes the corresponding coherent sheaf on $E$, and $\mathscr{G}_{P}=\mathscr{C}(\mathcal{M}, F)$ is what might be called the "characteristic sheaf" of the $\mathcal{D}$-module, defined on $P$. The support of the sheaf $\mathscr{G}_{E}$ is the characteristic variety of the $\mathcal{D}$-module $[1$, pp. 212-213]; it is a cone in $E$, and the support of $\mathscr{G}_{P}$ is the projectivization of that cone. Since $\mathcal{M}$ is holonomic, its characteristic variety is of pure dimension $d$. But because $M$ is a Hodge module, much more is true: in fact, Saito has shown that $\mathscr{G}$ is always a Cohen-Macaulay module over $\mathscr{S}$ (in [7, Lemme 5.1.13]). Consequently, the sheaf $\mathscr{G}_{E}$ is Cohen-Macaulay of dimension $d$ on $E$, and so Theorem 2.10 may be applied to it.

Moreover, the dual $\widehat{\mathscr{G}}=\mathcal{E}_{x} t_{\mathscr{S}}^{d}(\mathscr{G}, \omega \mathscr{S})=\mathcal{E}_{x} t_{\mathscr{S}}^{d}(\mathscr{G}, \mathscr{S}(-d)) \otimes \omega_{X}^{-1}$ can be computed explicitly in this case, since $M$ is polarized. To do this, recall from (1.2) that $\mathscr{G}^{\mathrm{r}}$ is the same graded $\mathscr{O}_{X}$-module as $\mathscr{G}$, but with the action of $\mathscr{S}$ changed so that sections of $\mathscr{S}_{k}$ act with an extra factor of $(-1)^{k}$. Evidently, we have $\mathscr{G}^{\mathrm{r}} \simeq \mathscr{G} \otimes \mathscr{S} \mathscr{S}^{\mathrm{r}}$.

Now let $M^{\prime}=\mathbb{D}_{X}(M)$ be the Verdier dual of the Hodge module, and $\left(\mathcal{M}^{\prime}, F\right)$ $=\mathbb{D}_{X}(\mathcal{M}, F)$ the underlying filtered left $\mathcal{D}$-module. According to [9, pp. 54-55], we have

$$
\mathcal{M}^{\prime}=\mathcal{E}_{x} t_{\mathcal{D}_{X}}^{d}\left(\mathcal{M}, \mathcal{D}_{X} \otimes_{\mathscr{O}_{X}} \omega_{X}^{-1}\right)
$$

where the filtration on $\mathcal{D}_{X} \otimes \omega_{X}^{-1}$ is given by $F_{p}\left(\mathcal{D}_{X} \otimes \omega_{X}^{-1}\right)=F_{p-2 d} \mathcal{D}_{X} \otimes \omega_{X}^{-1}$. Because of strictness (which is equivalent to the Cohen-Macaulay property), we can pass to the associated graded modules to obtain

$$
\operatorname{Gr}^{F} \mathcal{M}^{\prime}=\mathcal{E}_{x} t_{\mathscr{S}}^{d}\left(\mathscr{G}^{\mathrm{r}}, \mathscr{S}(-2 d)\right) \otimes \omega_{X}^{-1} \simeq \widehat{\mathscr{G}^{\mathrm{r}}}(-d)
$$

The change in module structure from $\mathscr{G}$ to $\mathscr{G}^{\mathrm{r}}$ happens because, in computing $\mathcal{M}^{\prime}$, one is really passing from $\mathcal{M}$ to the associated right $\mathcal{D}$-module $\omega_{X} \otimes_{\mathscr{O}_{X}} \mathcal{M}$, and the right action of $\Theta_{X}$ on $\omega_{X} \otimes \mathcal{M}$ is given by the rule

$$
(\omega \otimes m) \cdot \xi=(\omega \cdot \xi) \otimes m-\omega \otimes(\xi m),
$$

thus introducing an additional sign when passing to the graded module. 
A polarization on $M$ is an isomorphism $M \simeq \mathbb{D}_{X}(M)(-w)$, where $w$ is the weight of $M$. If $M$ is polarized, we thus have

$$
(\mathcal{M}, F) \simeq \mathbb{D}_{X}(\mathcal{M}, F)(-w)=\left(\mathcal{M}^{\prime}, F_{\bullet+w}\right)
$$

When combined with the isomorphism above, this gives

$$
\mathscr{G} \simeq\left(\operatorname{Gr}^{F} \mathcal{M}^{\prime}\right)(w) \simeq \widehat{\mathscr{G} \mathrm{r}}(w-d),
$$

or in other words, $\widehat{\mathscr{G}} \simeq \mathscr{G}^{\mathrm{r}}(d-w)$.

\section{§3.2. Duality for polarized Hodge modules}

We now obtain from Theorem 2.10 the following result about polarized Hodge modules.

Theorem 3.2. Let $M$ be a polarized Hodge module of weight $w=d+n$ on the nonsingular d-dimensional complex algebraic variety $X$. Let $(\mathcal{M}, F)$ be the underlying filtered left $\mathcal{D}$-module, and write $\mathscr{G}=\mathrm{Gr}^{F} \mathcal{M}$ for the associated graded $\mathscr{S}$-module. Also let $\mathscr{C}=\mathscr{C}(\mathcal{M}, F)$ be the corresponding coherent sheaf on $P=\mathbb{P}\left(\Theta_{X}\right)$. Then there is an exact sequence

$$
0 \rightarrow D\left(\mathscr{G}^{\mathrm{r}}(-n)\right) \rightarrow \mathscr{G} \rightarrow \Gamma_{*}(\mathscr{C}) \rightarrow D^{1}\left(\mathscr{G}^{\mathrm{r}}(-n)\right) \rightarrow 0
$$

of graded $\mathscr{S}$-modules on $X$. Similarly, for each $i \geq 2$, we have an isomorphism

$$
R^{i-1} \Gamma_{*}(\mathscr{C}) \simeq D^{i}\left(\mathscr{G}^{\mathrm{r}}(-n)\right)
$$

of graded $\mathscr{S}$-modules.

The graded $\mathscr{S}$-module $D^{i}\left(\mathscr{G}^{\mathrm{r}}(-n)\right)$ is easily described. Indeed, for any integer $k$, its graded piece in degree $k$ is

$$
D^{i}\left(\mathscr{G}^{\mathrm{r}}(-n)\right)_{k}=\mathcal{E} x t_{\mathscr{O}_{X}}^{i}\left(\mathscr{G}_{-n-k}, \mathscr{O}_{X}\right)=\mathcal{E}_{x} t_{\mathscr{O}_{X}}^{i}\left(\operatorname{Gr}_{-n-k}^{F} \mathcal{M}, \mathscr{O}_{X}\right) .
$$

We thus get, for each $k$, an exact sequence

$$
0 \rightarrow \mathcal{H o m}\left(\mathscr{G}_{-n-k}, \mathscr{O}_{X}\right) \rightarrow \mathscr{G}_{k} \rightarrow \pi_{*}\left(\mathscr{C} \otimes \mathscr{O}_{P}(k)\right) \rightarrow \mathcal{E} x t^{1}\left(\mathscr{G}_{-n-k}, \mathscr{O}_{X}\right) \rightarrow 0
$$

\section{$\S 3.3$. The role of the polarization}

The derivation of Theorem 3.2 shows that the map $D\left(\mathscr{G}^{\mathrm{r}}(-n)\right) \rightarrow \mathscr{G}$ in the exact sequence (3.3) is induced by the polarization of the Hodge module; in fact, the isomorphism $M \simeq \mathbb{D}_{X}(M)(-w)$ is exactly what was used to pass from Proposition 2.5 to Theorem 3.2. To see this more clearly, we first consider the case when $M$ comes from a polarized variation of Hodge structure. 
So let $\left(L_{\mathscr{O}}, \nabla, F, L_{\mathbb{Q}}, S\right)$ be a polarized variation of Hodge structure of weight $n$. The flat connection $\nabla$ makes the vector bundle $L_{\mathscr{O}}$ into a left $\mathcal{D}$-module, which we denote by $\mathcal{M}$; it is filtered by setting $F_{k} \mathcal{M}=F^{-k} L_{\mathscr{O}}$, because of Griffiths transversality. Note that the graded $\mathscr{S}$-module $\mathscr{G}=\operatorname{Gr}^{F} \mathcal{M}$ is of finite length.

Now consider the polarization $S: L_{\mathbb{Q}} \otimes L_{\mathbb{Q}} \rightarrow \mathbb{Q}(-n)$ of the variation. By definition, we have $S\left(F^{p} L_{\mathscr{O}}, F^{q} L_{\mathscr{O}}\right)=0$ for $p+q>n$; thus $S$ descends to a nondegenerate bilinear pairing between $\mathrm{Gr}_{F}^{-k} L_{\mathscr{O}}$ and $\operatorname{Gr}_{F}^{n+k} L_{\mathscr{O}}$ for all $k$. We get an isomorphism

$$
\bigoplus_{k \in \mathbb{Z}} \operatorname{Gr}_{k}^{F} \mathcal{M} \simeq \bigoplus_{k \in \mathbb{Z}} \mathcal{H o m}_{\mathscr{O}_{X}}\left(\operatorname{Gr}_{-n-k}^{F} \mathcal{M}, \mathscr{O}_{X}\right)
$$

Moreover, $S$ is flat for the connection $\nabla$, and so

$$
d S\left(\lambda_{1}, \lambda_{2}\right)=S\left(\nabla \lambda_{1}, \lambda_{2}\right)+S\left(\lambda_{1}, \nabla \lambda_{2}\right)
$$

for all sections $\lambda_{1}, \lambda_{2}$ of $L_{\mathscr{O}}$. When $\lambda_{1}$ is a section of $\operatorname{Gr}_{k-1}^{F} \mathcal{M}=\operatorname{Gr}_{F}^{-k+1} L_{\mathscr{O}}$, and $\lambda_{2}$ a section of $\operatorname{Gr}_{-n-k}^{F} \mathcal{M}=\operatorname{Gr}_{F}^{n+k} L_{\mathscr{O}}$, we therefore have

$$
0=\xi \cdot S\left(\lambda_{1}, \lambda_{2}\right)=S\left(\xi \cdot \lambda_{1}, \lambda_{2}\right)+S\left(\lambda_{1}, \xi \cdot \lambda_{2}\right)
$$

for arbitrary vector fields $\xi$. This shows that (3.5) is compatible with the action by $\mathscr{S}$, provided that sections of $\mathscr{S}_{k}$ act on the right-hand side with an extra factor of $(-1)^{k}$. In the notation used in $\S 3.1$, the polarization therefore determines an isomorphism of graded $\mathscr{S}$-modules $\mathscr{G} \simeq D\left(\mathscr{G}^{\mathrm{r}}(-n)\right)$.

Let $M$ be the polarized Hodge module associated to the variation [7, Theorem 5.4.3]; its weight is $w=d+n$. As expected, the map in (3.3) is the one given by the polarization $S$, up to a sign factor.

Lemma 3.6. Let $M$ be the Hodge module associated to a polarized variation of Hodge structure $\left(L_{\mathscr{O}}, \nabla, F, L_{\mathbb{Q}}, S\right)$ of weight $n$, with $S: L_{\mathbb{Q}} \otimes L_{\mathbb{Q}} \rightarrow \mathbb{Q}(-n)$ the polarization. Then the map $D\left(\mathscr{G}^{\mathrm{r}}(-n)\right) \rightarrow \mathscr{G}$ in $(3.3)$ is an isomorphism, whose inverse

$$
\bigoplus_{k \in \mathbb{Z}} \mathrm{Gr}_{F}^{k} L_{\mathscr{O}} \rightarrow \bigoplus_{k \in \mathbb{Z}} \mathcal{H o m}_{\mathscr{O}_{X}}\left(\mathrm{Gr}_{F}^{n-k} L_{\mathscr{O}}, \mathscr{O}_{X}\right)
$$

is given (up to a sign factor) by the formula $\lambda \mapsto S(\lambda,-)$ for $\lambda \in \operatorname{Gr}_{F}^{k} L_{\mathscr{O}}$.

The exact value of the sign depends on the sign conventions that are used for shifts, total complexes, etc., and so we shall ignore this point here. The proof of the lemma will be given in $\S 3.4$.

Now we return to the case of a general polarized Hodge module $M$ of weight $w=d+n$. There is always a dense Zariski-open subset $U \subseteq X$ on which $M$ is the Hodge module associated to a polarized variation of Hodge structure of weight $n$ 
[7, Lemme 5.1.10]. Over $U$, therefore, the map $D\left(\mathscr{G}^{\mathrm{r}}(-n)\right) \rightarrow \mathscr{G}$ is the one in Lemma 3.6. But then the same has to be true on all of $X$ by continuity. In other words, through the first map in (3.4), a local section $\sigma$ of $\mathcal{H o m}\left(\mathrm{Gr}_{-n-k}^{F} \mathcal{M}, \mathscr{O}_{X}\right)$ determines a local section $i(\sigma)$ of $\operatorname{Gr}_{k}^{F} \mathcal{M}$. Lemma 3.6 shows that, at least at points of $U$, we have

$$
\pm S(i(\sigma), m)=\sigma(m)
$$

for every local section $m$ of $\operatorname{Gr}_{-n-k}^{F} \mathcal{M}$. But since both sides are holomorphic functions, and $U$ is dense in $X$, the identity has to hold at points of $X-U$ as well.

Remark. A different way to think about this is the following: Over $U$, any section of $\mathrm{Gr}_{k}^{F} \mathcal{M}$ determines a linear functional on $\mathrm{Gr}_{-n-k}^{F} \mathcal{M}$. We can thus think of the sheaf $\pi_{*}\left(\mathscr{C}(\mathcal{M}, F) \otimes \mathscr{O}_{P}(k)\right)$ in (3.4), whose support is contained in the complement of $U$, as giving the obstructions for that functional to extend over points of $X-U$.

\section{§3.4. Proof of the lemma}

The first assertion in Lemma 3.6 is very easy to prove. Indeed, the characteristic variety of $\mathcal{M}$ is the zero section, and $\mathscr{G}$ has finite length as an $\mathscr{S}$-module. We thus have $\mathscr{C}(\mathcal{M}, F)=0$, and so the map $D(\widehat{\mathscr{G}}) \rightarrow \mathscr{G}$ in Theorem 2.10 is an isomorphism in this case. It follows that the first map in (3.3) is also an isomorphism. Now, given Saito's description of the polarization in [7, Lemme 5.4.2], it is certainly believable that the isomorphism should be given up to a sign by $S$ as in (3.5); however, it seemed advantageous to write down a more detailed proof. This is the purpose of the present section; it involves looking more closely at Saito's construction.

Since it becomes necessary to use both left and right $\mathcal{D}$-modules here, we shall introduce the following notation. As in $\S 3.3$, the filtered left $\mathcal{D}$-module determined by $\left(L_{\mathscr{O}}, \nabla, F\right)$ will be denoted by $(\mathcal{M}, F)$, and the associated graded $\mathscr{S}$-module by $\mathscr{G}=\operatorname{Gr}^{F} \mathcal{M}$. The corresponding right $\mathcal{D}$-module is then $\mathcal{N}=\omega_{X} \otimes_{\mathscr{O}_{X}} \mathcal{M}$, with $\mathcal{D}$-module structure defined by the rule

$$
(\omega \otimes m) \cdot \xi=(\omega \cdot \xi) \otimes m-\omega \otimes(\xi \cdot m)
$$

for $\xi$ any section of $\Theta_{X}$. The filtration is given by $F_{p} \mathcal{N}=\omega_{X} \otimes F_{p+d} \mathcal{M}$; together with (3.7), this shows that

$$
\operatorname{Gr}^{F} \mathcal{N} \simeq \omega_{X} \otimes_{\mathscr{O}_{X}} \mathscr{G}^{\mathrm{r}}(d)
$$

in the notation of $\S 3.1$.

Saito proves that $(\mathcal{N}, F)$ has a canonical resolution by induced $\mathcal{D}$-modules $[7$, Lemme 2.1.6]. It is constructed by taking the Spencer complex $\widetilde{\mathrm{DR}}(\mathcal{N}, F)$ (isomorphic to the de $\operatorname{Rham}$ complex $\operatorname{DR}(\mathcal{M}, F)$ for the original left $\mathcal{D}$-module), and 
tensoring on the right by $\left(\mathcal{D}_{X}, F\right)$; the augmentation map

$$
\widetilde{\operatorname{DR}}(\mathcal{N}, F) \otimes_{\mathscr{O}_{X}}\left(\mathcal{D}_{X}, F\right) \rightarrow(\mathcal{N}, F)
$$

is a filtered quasi-isomorphism. The associated graded complex

$$
\operatorname{Gr}^{F}\left(\widetilde{\mathrm{DR}}(\mathcal{N}, F) \otimes_{\mathscr{O}_{X}}\left(\mathcal{D}_{X}, F\right)\right)
$$

then provides a canonical resolution of $\operatorname{Gr}^{F} \mathcal{N}$ by basic graded $\mathscr{S}$-modules (as in Lemma 2.6), because $\operatorname{Gr}^{F} \mathcal{N}$ is locally free over $\mathscr{O}_{X}$ in our case. We let

$$
\mathscr{F}^{\bullet}=\omega_{X}^{-1} \otimes_{\mathscr{O}_{X}} \operatorname{Gr}^{F}\left(\widetilde{\operatorname{DR}}(\mathcal{N}, F) \otimes_{\mathscr{O}_{X}}\left(\mathcal{D}_{X}, F\right)\right)(-d),
$$

which resolves $\mathscr{G}^{\mathrm{r}}$ by basic graded $\mathscr{S}$-modules.

Saito's construction of the isomorphism $(\mathcal{M}, F) \simeq \mathbb{D}_{X}(\mathcal{M}, F)(-w)$ is the following. He shows that $S$ gives a filtered quasi-isomorphism

$$
\widetilde{\operatorname{DR}}(\mathcal{N}, F) \rightarrow \mathcal{H}_{o} m_{\mathscr{O}_{X}}^{F}\left(\widetilde{\mathrm{DR}}(\mathcal{N}, F),\left(\omega_{X}, F\right)[d]\right)(-w)
$$

note that, in this case only, the filtration on $\omega_{X}$ is such that $\operatorname{Gr}_{k}^{F} \omega_{X}=0$ for $k \neq 0$. Passing to induced modules, one gets a filtered quasi-isomorphism

$$
\widetilde{\mathrm{DR}}(\mathcal{N}, F) \otimes\left(\mathcal{D}_{X}, F\right) \rightarrow \mathcal{H}^{\prime} m_{\mathcal{D}_{X}}\left(\widetilde{\mathrm{DR}}(\mathcal{N}, F) \otimes\left(\mathcal{D}_{X}, F\right),\left(\omega_{X} \otimes \mathcal{D}_{X}, F\right)[d]\right)(-w)
$$

Here $\omega_{X} \otimes_{\mathscr{O}_{X}} \mathcal{D}_{X}$ has two different structures as a right $\mathcal{D}$-module; one is used when applying $\mathcal{H}^{\circ} m_{\mathcal{D}_{X}}(-,-)$, and the other to make the right-hand side into a complex of right $\mathcal{D}$-modules. Since $\operatorname{Gr}^{F} \mathcal{N}$ is locally free over $\mathscr{O}_{X}$, that complex computes the Verdier dual $\mathbb{D}_{X}(\mathcal{N}, F)(-w)$; seeing that the left-hand side is quasiisomorphic to $(\mathcal{N}, F)$, one has the desired polarization, on the level of filtered right $\mathcal{D}$-modules.

Using the strictness property of the right-hand side (again because $\mathscr{G}$ is Cohen-Macaulay), we can now pass to the associated graded complexes. Noting that a Tate twist operates by $(\mathcal{M}, F)(-w)=\left(\mathcal{M}, F_{\bullet+w}\right)$, we obtain a quasiisomorphism

$$
\omega_{X} \otimes \mathscr{F}^{\bullet}(d) \rightarrow \mathcal{H}^{\prime} m_{\mathscr{S}}\left(\omega_{X} \otimes \mathscr{F}^{\bullet}(d), \omega_{X} \otimes \mathscr{S}(w)[d]\right) \otimes \mathscr{S} \mathscr{S}^{\mathrm{r}}
$$

the change in module structure by $\mathscr{S}^{\mathrm{r}}$ is due to the difference between the two $\mathcal{D}$-module structures on $\omega_{X} \otimes_{\mathscr{O}_{X}} \mathcal{D}_{X}$. After some cancellation, and with the abbreviation $\omega_{\mathscr{S}}=\omega_{X}^{-1} \otimes \mathscr{S}(-d)$, we see that

$$
\mathscr{F}^{\bullet} \otimes \mathscr{S} \mathscr{S}^{\mathrm{r}} \rightarrow \mathcal{H o m}_{\mathscr{S}}\left(\mathscr{F}^{\bullet}(-n), \omega_{\mathscr{S}}[d]\right)
$$

is also a quasi-isomorphism. Note that it is still induced by $S$, up to a sign. 
As we observed before, the complex $\mathscr{F} \bullet \otimes \mathscr{S}^{\mathrm{r}}$ on the left-hand side is a resolution of $\mathscr{G}$ by basic graded $\mathscr{S}$-modules. Thus the complex

$$
D\left(\mathcal{H o m}_{\mathscr{S}}\left(\mathscr{F}^{\bullet} \otimes_{\mathscr{S}} \mathscr{S}^{\mathrm{r}}, \omega_{\mathscr{S}}[d]\right)\right)
$$

is quasi-isomorphic to $f_{*} \mathcal{H}_{X}^{0}\left(\mathscr{G}_{E}\right) \simeq \mathscr{G}$ by local duality, as in $\S 2.2$. On the other hand, it computes the $\mathscr{S}$-module $D(\widehat{\mathscr{G}})$, and the isomorphism $D(\widehat{\mathscr{G}}) \simeq \mathscr{G}$ in Proposition 2.5 is therefore directly given by that complex.

Returning to (3.8), we find that

$$
D\left(\mathcal{H}_{o} m_{\mathscr{S}}\left(\mathscr{F}^{\bullet} \otimes_{\mathscr{S}} \mathscr{S}^{\mathrm{r}}, \omega_{\mathscr{S}}[d]\right)\right) \simeq D\left(\mathscr{F}^{\bullet}(-n)\right) \simeq D\left(\mathscr{G}^{\mathrm{r}}(-n)\right),
$$

since $\mathscr{F}^{\bullet}$ resolves $\mathscr{G}^{\mathrm{r}}$. If we compose this isomorphism with the inverse of $D(\widehat{\mathscr{G}}) \simeq \mathscr{G}$, we obtain a map

$$
\mathscr{G} \rightarrow D\left(\mathscr{G}^{\mathrm{r}}(-n)\right)
$$

by construction, it is the inverse of the isomorphism in (3.3). On the other hand, our derivation shows that it is given by $\pm S$, and so the remaining assertion of Lemma 3.6 is proved.

\section{$\S 4$. Several consequences}

\section{$\S 4.1$. Derived category formulation}

When applying other functors, it is more convenient to have a version of (3.3) in the derived category; such a version is easily deduced from $§ 2.1-3.1$.

Throughout, we will employ the following (mostly standard) notation for derived categories and derived functors. We let $\mathrm{D}_{\mathrm{qc}}^{\mathrm{b}}\left(\mathscr{O}_{X}\right)$ be the bounded derived category of quasi-coherent sheaves on $X$, and $\mathrm{D}^{\mathrm{b}}\left(\mathscr{O}_{X}\right)$ the full subcategory of objects whose cohomology sheaves are coherent. Similarly, we write $\mathrm{D}_{\mathrm{qc}, \mathrm{gr}}^{\mathrm{b}}(\mathscr{S})$ for the bounded derived category of quasi-coherent and graded $\mathscr{S}$-modules, and $\mathrm{D}_{\mathrm{gr}}^{\mathrm{b}}(\mathscr{S})$ for the full subcategory of objects whose cohomology sheaves are finitely generated as $\mathscr{S}$-modules. As already mentioned, there is an equivalence of categories

$$
\mathbb{R} f_{*}: \mathrm{D}_{\mathrm{qc}}^{\mathrm{b}}\left(\mathscr{O}_{E}\right) \rightarrow \mathrm{D}_{\mathrm{qc}}^{\mathrm{b}}(\mathscr{S})
$$

for an object $F \in \mathrm{D}_{\mathrm{qc}}^{\mathrm{b}}(\mathscr{S})$, we write $F_{E}$ for the corresponding object in $\mathrm{D}_{\mathrm{qc}}^{\mathrm{b}}\left(\mathscr{O}_{E}\right)$ such that $\mathbb{R} f_{*} F_{E} \simeq F$. Similarly, for $G \in \mathrm{D}_{\mathrm{qc}, \mathrm{gr}}^{\mathrm{b}}(\mathscr{S})$, we let $G_{P}$ be the corresponding object in $\mathrm{D}_{\mathrm{qc}}^{\mathrm{b}}\left(\mathscr{O}_{P}\right)$. Both operations are exact functors.

The symbol $\mathcal{H}^{i}(-)$ means the $i$-th cohomology sheaf of a complex of sheaves. The derived functor of the tensor product will be denoted by $\underline{\otimes}$. We write

$$
\mathbb{L} D: \mathrm{D}_{\mathrm{qc}, \mathrm{gr}}^{\mathrm{b}}(\mathscr{S}) \rightarrow \mathrm{D}_{\mathrm{qc}, \mathrm{gr}}^{\mathrm{b}}(\mathscr{S})^{\mathrm{op}}
$$


for the derived functor of the graded dual in (2.3); we also let

$$
\mathbb{R} \Gamma_{*}: \mathrm{D}^{\mathrm{b}}\left(\mathscr{O}_{P}\right) \rightarrow \mathrm{D}_{\mathrm{gr}}^{\mathrm{b}}(\mathscr{S})
$$

be the derived functor of $\mathscr{F} \mapsto \Gamma_{*}(\mathscr{F})=\bigoplus_{k \in \mathbb{Z}} \mathscr{F} \otimes \mathscr{O}_{P}(k)$.

The results about local cohomology and local duality from $\S 2.1-2.2$ are easily translated into the language of derived categories. To begin with, we have the following restatement of Proposition 2.1.

Lemma 4.1. For any object $G \in \mathrm{D}_{\mathrm{gr}}^{\mathrm{b}}(\mathscr{S})$, there is a functorial exact triangle

$$
\mathbb{R} f_{*} \mathbb{R} \mathcal{H}_{X}^{0}\left(G_{E}\right) \rightarrow G \rightarrow \mathbb{R} \Gamma_{*}\left(G_{P}\right) \rightarrow \mathbb{R} f_{*} \mathbb{R} \mathcal{H}_{X}^{0}\left(G_{E}\right)[1]
$$

in the derived category $\mathrm{D}_{\mathrm{qc}, \mathrm{gr}}^{\mathrm{b}}(\mathscr{S})$ of graded, quasi-coherent $\mathscr{S}$-modules on $X$.

Proof. The result from [5, p. 16] that was used in the proof of Proposition 2.1 is based on the exact triangle

$$
\mathbb{R} \mathcal{H}_{X}^{0}\left(G_{E}\right) \rightarrow G_{E} \rightarrow \mathbb{R} j_{*} j^{*} G_{E} \rightarrow \mathbb{R} \mathcal{H}_{X}^{0}\left(G_{E}\right)[1]
$$

in the derived category $\mathrm{D}_{\mathrm{qc}}^{\mathrm{b}}\left(\mathscr{O}_{E}\right)$ of quasi-coherent sheaves on $E$. To get the conclusion, simply apply the functor $\mathbb{R} f_{*}$ to that triangle, and then argue as before.

In like manner, local duality from Proposition 2.5 can be reformulated as follows. Note that this only works for finitely generated $\mathscr{S}$-modules, because of the necessity of resolving by basic $\mathscr{S}$-modules.

Lemma 4.2. For any object $G \in \mathrm{D}_{\mathrm{gr}}^{\mathrm{b}}(\mathscr{S})$, there is a functorial isomorphism

$$
\mathbb{R} f_{*} \mathbb{R} \mathcal{H}_{X}^{0}\left(G_{E}\right) \simeq \mathbb{L} D \mathbb{R} \mathcal{H}_{\mathscr{S}}\left(G, \omega_{\mathscr{S}}[d]\right)
$$

in $\mathrm{D}_{\mathrm{qc}, \mathrm{gr}}^{\mathrm{b}}(\mathscr{S})$.

By combining the two lemmas with the arguments from $\S 2.3-3.1$, we arrive at the following derived-category version of Theorem 3.2.

Theorem 4.3. With the assumptions and the notation of Theorem 3.2, there is an exact triangle

$$
\mathbb{L} D\left(\mathscr{G}^{\mathrm{r}}(-n)\right) \rightarrow \mathscr{G} \rightarrow \mathbb{R} \Gamma_{*}(\mathscr{C}(\mathcal{M}, F)) \rightarrow \mathbb{L} D\left(\mathscr{G}^{\mathrm{r}}(-n)\right)[1]
$$

in $\mathrm{D}_{\mathrm{qc}, \mathrm{gr}}^{\mathrm{b}}(\mathscr{S})$. 


\section{$\S 4.2$. Graded de Rham complexes}

We now wish to apply Theorem 4.3 to the study of the de Rham complex $\operatorname{DR}_{X}(\mathcal{M})$ of a filtered $\mathcal{D}$-module $(\mathcal{M}, F)$ underlying a polarized Hodge module. Since our result gives information about the associated graded complex, we begin by proving several simple lemmas about the de Rham complex for general graded $\mathscr{S}$-modules.

Given any graded $\mathscr{S}$-module $\mathscr{F}$, we can form the Koszul complex for the multiplication map $\Theta_{X} \otimes_{\mathscr{O}_{X}} \mathscr{F} \rightarrow \mathscr{F}(1)$, and tensor by $\omega_{X}$, to arrive at the Koszul-type complex

$$
\operatorname{DR}(\mathscr{F})=\left[\mathscr{F} \rightarrow \Omega_{X}^{1} \otimes \mathscr{F}(1) \rightarrow \Omega_{X}^{2} \otimes \mathscr{F}(2)-\cdots \rightarrow \Omega_{X}^{d} \otimes \mathscr{F}(d)\right][d]
$$

of graded $\mathscr{S}$-modules. We call this the de Rham complex for the graded module $\mathscr{F}$; as commonly done, we put it in degrees $-d, \ldots, 0$, as indicated by the shift. Obviously, we have $\operatorname{DR}(\mathscr{F}) \simeq \operatorname{DR}(\mathscr{S}) \otimes_{\mathscr{S}} \mathscr{F}$. Since $\mathscr{S}=\operatorname{Sym} \Theta_{X}$, the complex $\operatorname{DR}(\mathscr{S})$ is a free resolution of $\omega_{X}(d)=\omega_{X} \otimes \mathscr{S} \mathscr{S}(d)$, where $\omega_{X}$ has the trivial $\mathscr{S}$-module structure; we therefore have $\mathrm{DR}(\mathscr{S}) \simeq \omega_{X}(d)$ in $\mathrm{D}_{\mathrm{gr}}^{\mathrm{b}}(\mathscr{S})$. It follows that $\mathscr{F} \mapsto \mathrm{DR}(\mathscr{F}) \simeq \mathrm{DR}(\mathscr{S}) \otimes \mathscr{S} \mathscr{F} \simeq \omega_{X} \otimes_{\mathscr{S}} \mathscr{F}(d)$ gives rise to an exact functor

$$
\mathrm{DR}: \mathrm{D}_{\mathrm{qc}, \mathrm{gr}}^{\mathrm{b}}(\mathscr{S}) \rightarrow \mathrm{D}_{\mathrm{qc}, \mathrm{gr}}^{\mathrm{b}}(\mathscr{S}) \text {. }
$$

A simple, but useful observation is that the cohomology sheaves of the de Rham complex are always of finite length as $\mathscr{S}$-modules.

Lemma 4.4. Let $G \in \mathrm{D}_{\mathrm{qc}, \mathrm{gr}}^{\mathrm{b}}(\mathscr{S})$ be any object. Then $\mathrm{DR}(G)_{P} \simeq 0$ in $\mathrm{D}_{\mathrm{qc}}^{\mathrm{b}}\left(\mathscr{O}_{P}\right)$.

Proof. As an $\mathscr{S}$-module, $\omega_{X}(d)$ is torsion, and so its associated coherent sheaf on $P$ is zero. Thus we also have $\operatorname{DR}(\mathscr{S})_{P} \simeq 0$. Alternatively, one can consider the Euler sequence

$$
0 \rightarrow \mathscr{O}_{P} \rightarrow \pi^{*} \Omega_{X}^{1} \otimes \mathscr{O}_{P}(1) \rightarrow \Theta_{P / X} \rightarrow 0
$$

on the projectivized cotangent bundle $\pi: P \rightarrow X$, and observe that its $d$-th wedge product gives an exact complex

$$
\left[\mathscr{O}_{P} \rightarrow \pi^{*} \Omega_{X}^{1} \otimes \mathscr{O}_{P}(1) \rightarrow \pi^{*} \Omega_{X}^{2} \otimes \mathscr{O}_{P}(2)-\cdots \rightarrow \pi^{*} \Omega_{X}^{d} \otimes \mathscr{O}_{P}(d)\right] .
$$

It quickly follows that $\operatorname{DR}(\mathscr{S})_{P}$ is also exact, and therefore isomorphic to zero in $\mathrm{D}^{\mathrm{b}}\left(\mathscr{O}_{P}\right)$. Either way, we then have

$$
\operatorname{DR}(G)_{P} \simeq\left(\operatorname{DR}(\mathscr{S}) \underline{\otimes}_{\mathscr{S}} G\right)_{P} \simeq \operatorname{DR}(\mathscr{S})_{P} \underline{\otimes}_{\mathscr{O}_{P}} G_{P} \simeq 0,
$$

because the operation $(-)_{P}$ is compatible with tensor products. 
The results of $\S 4.1$ take a very simple form when applied to a de Rham complex.

Lemma 4.5. For any object $G \in \mathrm{D}_{\mathrm{gr}}^{\mathrm{b}}(\mathscr{S})$, there are functorial isomorphisms

$$
\operatorname{DR}(\mathscr{S}) \underline{\otimes}_{\mathscr{S}} \mathbb{L} D \mathbb{R} \mathcal{H}_{\mathscr{S}} m_{\mathscr{S}}\left(G, \omega_{\mathscr{S}}[d]\right) \simeq \mathbb{L} D \mathbb{R} \mathcal{H}_{\mathscr{S}}\left(\operatorname{DR}(G), \omega_{\mathscr{S}}[d]\right) \simeq \operatorname{DR}(G) .
$$

Proof. Apply Lemma 4.1 to the object $\operatorname{DR}(G)$ to obtain an exact triangle

$$
\mathbb{R} f_{*} \mathbb{R} \mathcal{H}_{X}^{0}\left(\mathrm{DR}(G)_{E}\right) \rightarrow \mathrm{DR}(G) \rightarrow \mathbb{R} \Gamma_{*}\left(\mathrm{DR}(G)_{P}\right) \rightarrow \mathbb{R} f_{*} \mathbb{R} \mathcal{H}_{X}^{0}\left(\mathrm{DR}(G)_{E}\right)[1]
$$

whose third term is isomorphic to zero by Lemma 4.4. The triangle therefore degenerates to an isomorphism

$$
\mathbb{R} f_{*} \mathbb{R} \mathcal{H}_{X}^{0}\left(\mathrm{DR}(G)_{E}\right) \simeq \mathrm{DR}(G) .
$$

Lemma 4.2 now implies one half of the assertion. For the other, we note that

$$
\begin{aligned}
\mathbb{R} f_{*} \mathbb{R} \mathcal{H}_{X}^{0}\left(\operatorname{DR}(G)_{E}\right) & \simeq \mathbb{R} f_{*} \mathbb{R} \mathcal{H}_{X}^{0}\left(\operatorname{DR}(\mathscr{S})_{E} \underline{\otimes}_{\mathscr{O}_{E}} G_{E}\right) \\
& \simeq \operatorname{DR}(\mathscr{S}) \underline{\otimes}_{\mathscr{S}} \mathbb{R} f_{*} \mathbb{R} \mathcal{H}_{X}^{0}\left(G_{E}\right),
\end{aligned}
$$

and then conclude by invoking Lemma 4.2 a second time.

\section{§4.3. Properties of the de Rham complex}

After these preparations, we can now apply Theorem 3.2 to study the de Rham complex

$$
\mathrm{DR}_{X}(\mathcal{M})=\left[\mathcal{M} \rightarrow \Omega_{X}^{1} \otimes \mathcal{M} \rightarrow \Omega_{X}^{2} \otimes \mathcal{M}-\cdots \rightarrow \Omega_{X}^{d} \otimes \mathcal{M}\right][d]
$$

of the filtered $\mathcal{D}$-module $(\mathcal{M}, F)$. As in $\S 4.2$, the complex is supported in degrees $-d, \ldots, 0$. It is naturally filtered by subcomplexes

$$
F_{k} \mathrm{DR}_{X}(\mathcal{M})=\left[F_{k} \mathcal{M} \rightarrow \Omega_{X}^{1} \otimes F_{k+1} \mathcal{M}-\cdots \rightarrow \Omega_{X}^{d} \otimes F_{k+n} \mathcal{M}\right][d]
$$

moreover, $\operatorname{Gr}^{F} \operatorname{DR}_{X}(\mathcal{M})$ is a complex of finitely-generated graded $\mathscr{S}$-modules.

As a matter of fact, it is not hard to see that

$$
\operatorname{Gr}^{F} \operatorname{DR}_{X}(\mathcal{M}) \simeq \operatorname{DR}\left(\operatorname{Gr}^{F} \mathcal{M}\right)=\operatorname{DR}(\mathscr{G}) .
$$

We can therefore obtain information about the associated graded of the de Rham complex of $\mathcal{M}$ by applying Lemma 4.5 to the complex $\operatorname{DR}(\mathscr{G})$. When combined with Theorem 4.3, the following result emerges. 
Proposition 4.7. Let $M$ be a polarized Hodge module of weight $w=d+n$ on a nonsingular d-dimensional complex algebraic variety, let $(\mathcal{M}, F)$ be the underlying filtered left $\mathcal{D}$-module, and $\mathscr{G}=\mathrm{Gr}^{F} \mathcal{M}$ the associated graded $\mathscr{S}$-module. Let $\operatorname{DR}(\mathscr{G})=\operatorname{Gr}^{F} \operatorname{DR}_{X}(\mathcal{M})$ be the graded de Rham complex. Then

$$
\operatorname{DR}(\mathscr{S}) \underline{\otimes}_{\mathscr{S}} \mathbb{L} D\left(\mathscr{G}^{\mathrm{r}}(-n)\right) \simeq \operatorname{DR}(\mathscr{G})
$$

in the derived category $\mathrm{D}_{\mathrm{gr}}^{\mathrm{b}}(\mathscr{S})$ of graded, coherent $\mathscr{S}$-modules.

In particular, there is a convergent spectral sequence

$$
E_{1}^{p, q}=\left(\Omega_{X}^{p+d} \otimes_{\mathscr{O}_{X}} \mathscr{S}(p+d)\right) \otimes \mathscr{S} D^{q}\left(\mathscr{G}^{\mathrm{r}}(-n)\right) \Longrightarrow \mathcal{H}^{p+q}\left(\operatorname{Gr}^{F} \operatorname{DR}_{X}(\mathcal{M})\right)
$$

of graded $\mathscr{S}$-modules; explicitly, the degree $k$ part is

$$
\left(E_{1}^{p, q}\right)_{k}=\mathcal{E} x t_{\mathscr{O}_{X}}^{q}\left(\mathscr{G}_{-w-k-p}, \Omega_{X}^{p+d}\right) \Longrightarrow \mathcal{H}^{p+q}\left(\operatorname{Gr}_{k}^{F} \operatorname{DR}_{X}(\mathcal{M})\right) .
$$

The spectral sequence has a useful consequence for the de Rham complex of $\mathcal{M}$.

Proposition 4.8. Let $m \in \mathbb{Z}$ be such that $F_{-m} \mathcal{M}=0$. Then the inclusion $F_{m-n-1} \mathrm{DR}_{X}(\mathcal{M}) \subseteq \mathrm{DR}_{X}(\mathcal{M})$ is a filtered quasi-isomorphism.

Proof. By assumption, $\mathscr{G}_{k}=0$ for all $k \leq-m$; in the spectral sequence, we therefore have $\left(E_{1}^{p, q}\right)_{k}=0$ for all $p, q \in \mathbb{Z}$, provided that $-w-k+d=-n-k \leq-m$. This means that $\operatorname{Gr}_{k}^{F} \operatorname{DR}_{X}(\mathcal{M})$ is exact for $k \geq m-n$. Since $F_{\bullet} \mathcal{M}$ is a good filtration on $\mathcal{M}$, the assertion follows.

To see what this means, let us suppose that $M$ is the intermediate extension of a polarized variation of Hodge structure of weight $n$, which is "effective," meaning that $F^{n+1} \mathcal{M}=F_{-n-1} \mathcal{M}=0$. Then $F_{0} \mathrm{DR}_{X}(\mathcal{M}) \subseteq \mathrm{DR}_{X}(\mathcal{M})$ is a filtered quasi-isomorphism by the proposition. This implies, for instance, that any class in $\mathbb{H}^{-d+1}\left(\mathrm{DR}_{X}(\mathcal{M})\right)$ can be represented by a closed form $\omega \in \Gamma\left(X, \Omega_{X}^{1} \otimes F_{1} \mathcal{M}\right)$.

\section{Acknowledgements}

I thank Prof. Morihiko Saito for his careful reading of the paper.

\section{References}

[1] A. Borel, P.-P. Grivel, B. Kaup, A. Haefliger, B. Malgrange and F. Ehlers, Algebraic D-modules, Perspect. Math. 2, Academic Press, Boston, MA, 1987. Zbl 0642.32001 MR 0882000

[2] P. Brosnan, H. Fang, Z. Nie and G. Pearlstein, Singularities of admissible normal functions, Invent. Math. 177 (2009), 599-629. Zbl 1174.14009 MR 2534102 
[3] D. Eisenbud, The geometry of syzygies. A second course in commutative algebra and algebraic geometry, Grad. Texts in Math. 229, Springer, New York, 2005. Zbl 1066.14001 MR 2103875

[4] A. Grothendieck, Éléments de géométrie algébrique. II. Étude globale élémentaire de quelques classes de morphismes, Inst. Hautes Études Sci. Publ. Math. 8 (1961). Zbl 0118.36206 MR 0217084

[5] _ Cohomologie locale des faisceaux cohérents et théorèmes de Lefschetz locaux et globaux (SGA 2), Séminaire de Géométrie Algébrique du Bois-Marie, 1962. Zbl 0197.47202 MR 0476737

[6] M. Kashiwara and T. Kawai, The Poincaré lemma for a variation of polarized Hodge structure, Publ. Res. Inst. Math. Sci. 23 (1987), 345-407. Zbl 0629.14005 MR 0890924

[7] M. Saito, Modules de Hodge polarisables. Publ. Res. Inst. Math. Sci. 24 (1988), 849-995. Zbl 0691.14007 MR 1000123

[8] _ Mixed Hodge modules, Publ. Res. Inst. Math. Sci. 26 (1990), 221-333. Zbl 0691.14007 MR 1047415

[9] $\_$, On the theory of mixed Hodge modules, Amer. Math. Soc. Transl. (Ser. 2) 160 (1994), 47-61. Zbl 0815.14008 MR 1308540

[10] C. Schnell, The boundary behavior of cohomology classes and singularities of normal functions, Ph.D. thesis, Ohio State Univ., 2008. Available at OhioLink ETD. MR 2712093

[11] Complex analytic Néron models for arbitrary families of intermediate Jacobians, Invent. Math., to appear.

[12] — Residues and filtered $\mathcal{D}$-modules, Math. Ann., to appear. 